\title{
Electromagnetic Guided Percutaneous Renal Access Outcomes Among Surgeons and Trainees of Different Experience Levels: A Pilot Study
}

Michael S. Borofsky¹, Marcelino E. Rivera², Casey A. Dauw³ ${ }^{3}$, Amy E. Krambeck ${ }^{4}$, James E. Lingeman ${ }^{4}$

1. University of Minnesota, Minneapolis, MN. Electronic address: mborofsk@umn.edu.

2. Mayo Clinic, Rochester, MN.

3. University of Michigan, Ann Arbor, Ml.

4. Indiana University, Indianapolis, IN

This is the author's manuscript of the article published in final edited form as:

Borofsky, M. S., Rivera, M. E., Dauw, C. A., Krambeck, A. E., \& Lingeman, J. E. (2020). Electromagnetic Guided Percutaneous Renal Access Outcomes Among Surgeons and Trainees of Different Experience Levels: A Pilot Study. Urology, 136, 266-271. https://doi.org/10.1016/j.urology.2019.08.060 


\section{ABSTRACT}

\section{Objective}

To determine feasibility of an electromagnetic (EM) guidance system (Auris Health, Redwood City, CA) in obtaining percutaneous renal access among urologists and trainees of different experience levels. EM-guidance is appealing for access as it allows real time, 3-dimensional targeting without radiation. Few studies have explored this for percutaneous nephrolithotomy (PCNL) and none have assessed its potential to decrease the learning curve in obtaining access using traditional techniques.

\section{Methods}

Institutional Animal Care and Use Committee approval was obtained to compare EMguided percutaneous access to fluoroscopic guided access in a porcine model. Voluntary participants included urology trainees and faculty. They were categorized as beginner (no prior primary percutaneous nephrolithotomyexperience), intermediate (10-100 prior) and advanced (>100). Each participant attempted an EM and fluoroscopic guided puncture. Primary outcome was successful puncture. Secondary outcomes included access time, fluoroscopy time, and number of attempts. Participants were limited to 3 attempts and 10 minutes total to obtain access using each technique.

\section{Results}

Fourteen participants ( 6 beginners, 4 intermediates, and 4 experts) attempted 28 punctures. Overall success using EM-guidance was $93 \%$ compared to $71 \%$ using fluoroscopy $(P=.33)$. EM punctures had shorter access times (85 vs 255 seconds, $P<.01$ ) required fewer attempts ( 1 vs $2, P=.04$ ) and had decreased associated fluoroscopy times ( 1 vs 96 seconds, $P<.01$ ) excluding the initial retrograde pyelogram and guidance of the ureteroscope to the desired calyx. Beginners showed comparable success rates and outcomes relative to experts despite higher access times.

\section{Conclusion}

EM-guidance is a promising new technique to decrease the learning curve of percutaneous access with high success rates and minimal radiation. 
The ability to obtain one's own access is widely recognized to be a valuable skill for urologists who perform percutaneous nephrolithotomy (PCNL); yet, recent national estimates from the United States and United Kingdom show that radiologists, not urologists, are the ones obtaining renal access in the vast majority of cases. $1, \underline{2}$

One potential explanation as to why urologists do not obtain access more commonly is a steep learning curve with existing modalities used for this purpose (fluoroscopy and ultrasound). $\underline{3}, \underline{4}, \underline{5}, \underline{6}$ One promising technology that has yet to be applied widely to PCNL is electromagnetic (EM) guidance. EM-guidance is appealing for access as it allows real time, 3-dimensional targeting without any radiation.

To date, few studies have explored EM guidance as it relates to PCNL and no prior work has evaluated whether such technology can potentially decrease the learning curve in gaining access. We sought to determine the feasibility of a new EM guidance system (Auris Health, Redwood City, CA) in obtaining percutaneous access in a porcine model among urologists and trainees of different experience levels.

\section{METHODS}

Institutional Animal Care and Use Committee approval was obtained to perform the procedures on domestic farm pigs between 50 and $70 \mathrm{~kg}$ (Study Approval \# 2016.09).

\section{Procedure}

All pigs were prepared for percutaneous renal access by first performing cystoscopy to facilitate retrograde placement of ureteral access sheath to the proximal ureter on each side of the body. The pigs were then repositioned prone on the operating table with the ureteral access sheaths remaining accessible so that ureteroscopy could be performed to confirm placement of the needle in the collecting system. Prior to each percutaneous puncture a retrograde pyelogram was performed and fluoroscopy with a mobile C-Arm was used to help guide the ureteroscope into the desired calyx and confirm position. The amount of fluoroscopy time for this step was not included the in the total fluoroscopy time of the procedure. Fluoroscopy time for each puncture was measured and is reported specific to the percutaneous needle placement alone. 


\section{Fluoroscopic Guided Access (FG-access)}

After positioning the ureteroscope into a desired calyx, FG-access was attempted using an $18 \mathrm{~g}$ diamond tipped needle via either a triangulation based or bull's eye approach. Each participant was given up to 3 consecutive attempts to obtain successful access into a designated calyx, as confirmed by identification of the needle entering the collecting system visualized by the ureteroscope. Participants were limited to 10 minutes of total time in attempting access or 5 minutes of fluoroscopy time. Timing (procedure and fluoroscopy time) was started once the participant stated being ready to proceed, prior to activating the C-Arm. Failure was defined as inability to visually identify the needle in the collecting system with the ureteroscope when the participant anticipated it had reached the collecting system. In the event of failure, the needle was pulled back to the skin and the participant made a new attempt at gaining access.

\section{Electromagnetic Guided Access (EM-access)}

The EM guidance system consists of 4 specific components. First is an EM "targeting beacon" which is a 0.035 inch $(2.7 \mathrm{Fr}$ ) instrument passed through the working channel of the ureteroscope (Fig. 1). This provides a target for access that is able to be visualized on the graphical user interface (GUI) of the system. Second is a modified $18 \mathrm{~g}$ diamond tipped percutaneous access needle that contains an EM sensor built into the obturator. Each of these EM devices are able to be sensed by an EM field generator which is a $20 \times 20 \times 7 \mathrm{~cm}$ box-shaped device mounted device that can be connected to the bedside

rail and positioned outside the working field at the level of planned access. EM specific information regarding the relative position of the "beacon" and sensor is then depicted visually on the GUI (Fig. 2). The GUI provides targeting information including both relative direction (cranial and/or caudal, medial and/or lateral) of the needle and/or sensor relative to the target as well as the distance. The user is then able to adjust the position of the needle and/or sensor and receive real-time feedback regarding targeting via the GUI. Once the needle is appropriately lined up to reach the target in a straight pathway, the target as depicted on the GUI changes from red to green alerting the user that an appropriate needle orientation for access to the target has been identified. At this point 
the user advances the needle until the distance between the sensor and beacon becomes minimal or goes to 0 .

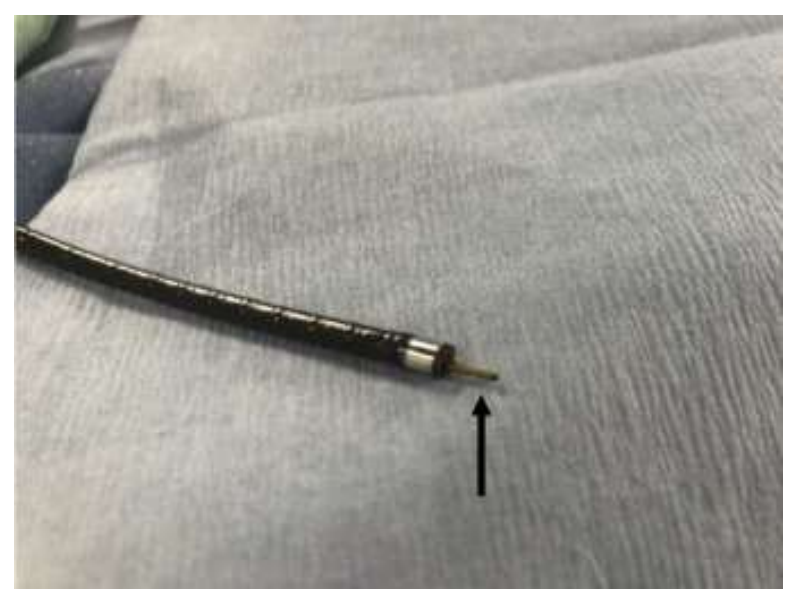

Figure 1. A 2.7 Fr electromagnetic targeting beacon as seen once passed through the working channel of a flexible ureteroscope. (Color version available online.)

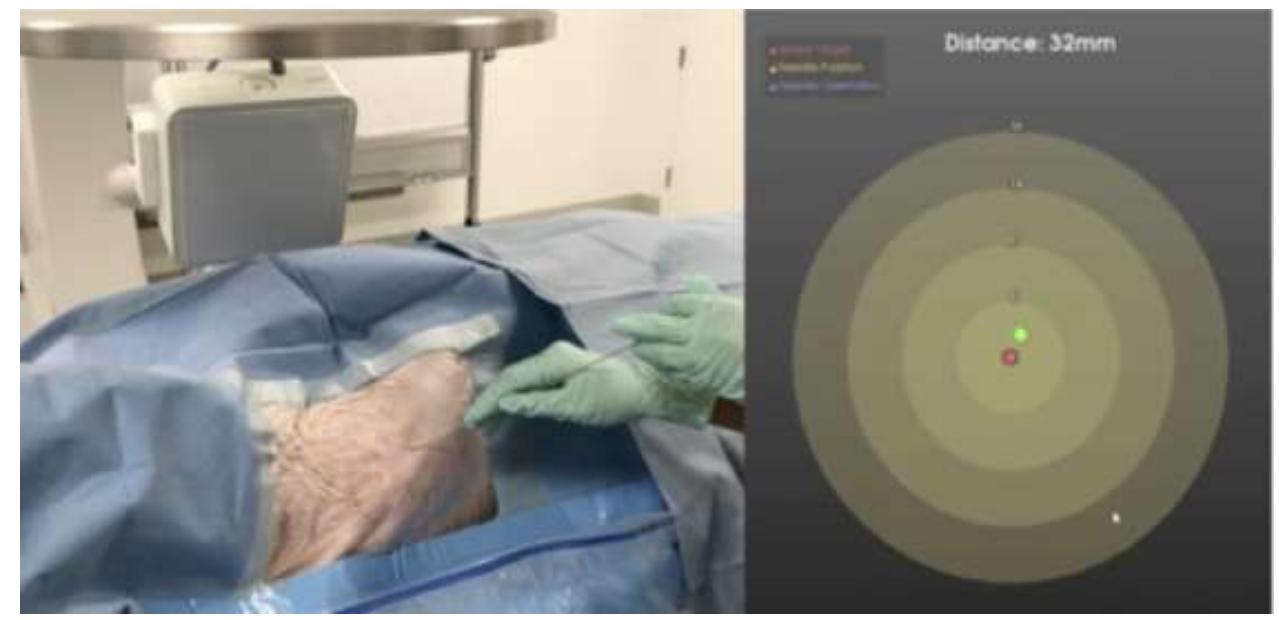

Figure 2. EM field generator is visualized as an attachment to the bedside rail. Modified percutaneous renal access needle with EM sensor built into obturator (Left). Graphic user interface depicting position of needle (green dot) relative to target (EM beacon, red dot) when positioned within the kidney. (Right). EM, electromagnetic. (Color version available online.)

EM-Access was attempted by each study participant. Prior to starting timing, retrograde ureteroscopy was performed to identify the desired calyx. Once the desired calyx was selected, the ureteroscope was held in place and the EM sensor was advanced through 
the scope so that it sat just in front of the papilla or in direct contact with it when feasible. As with FG-Access, fluoroscopy was used to guide the ureteroscope and confirm placement within the desired calyx. Once the participant indicating being ready to start the puncture, procedure, and fluoroscopy timing began. The participant used visual information provided by the GUI to align the needle with the target as depicted on the screen. Once the target became green, the participant advanced the needle until either the distance became zero or the needle could be seen entering the collecting system. As with FG-Access, each participant was given up to 3 consecutive attempts within a 10 minute time period to obtain successful access, as confirmed by identification of the needle entering the collecting system visualized by the ureteroscope. New attempts were defined using the same criteria as with fluoroscopy as well.

\section{Study Design}

Participants in the beginner cohort consisted of medical student subinterns on urology rotations $(n=3)$ as well as junior level residents $(n=3)$ who had not yet completed endourology rotations or participated in prior PCNLs as a primary surgeon. They were provided a lecture on percutaneous renal surgery and the concepts specific to the technical steps of obtaining FG-access. Intermediate level participants $(n=4)$ consisted of senior level residents and endourology fellows that had performed at least 10 prior PCNL accesses. Median number of prior PCNL access was 30 based on self-reported estimates (range 15-75). Expert surgeons consisted of practicing endourologists with fellowship training who had each performed 150 or more prior PCNL accesses (median 575 , range 150-15000).

Each participant performed an EM guided and fluoroscopy guided puncture the order of which was flipped with each subsequent participant. Each calyx was used only 1 time for access. Primary outcome was measured as success as determined by direct visualization of the needle entering the kidney with a ureteroscope. Secondary outcomes included access time, fluoroscopy time, and number of attempts. Statistical analysis was performed using JMP 14.1 software. Fischer exact testing was used to determine statistical significance for categorical data and nonparametric Mann-Whitney $U$ test was used to determine statistical significance for continuous data. The study was sponsored 
by Auris Health, the manufacturer of the EM technology. The authors retained full control of the design of the study, methods used, outcome parameters and results, analysis of data and production of the manuscript.

\section{RESULTS}

Fourteen participants attempted percutaneous renal access (6 beginners, 4 intermediates, and 4 experts) leading to 28 attempts in all. EM-access was successfully obtained in $93 \%(13 / 14)$ of attempts compared to FG-access where success was achieved in $71 \%(10 / 14), P=.326$.

Overall and by the skill level, there were fewer attempts prior to successful access with EM-Access compared to FG-Access (Table 1). In the EM group, $79 \%(11 / 14)$ of cases resulted in obtaining the access with a single attempt; in the FG group, 36\% (5/14) of cases resulted in access with a single attempt, $P=.06$. The biggest observed difference was in the beginner group; the EM-Access was obtained successfully with a single attempt in $100 \%(6 / 6)$ cases vs $33 \%(2 / 6)$ with the FG-Access, $P=.061$.

Table 1. Performance comparison between electromagnetic (EM) and fluoroscopic (FG) guided access

\begin{tabular}{|c|c|c|c|}
\hline & EM-Access & FG-Access & $P$ Value \\
\hline Success Rate & $13 / 14(93)$ & $10 / 14(71)$ & .326 \\
\hline Beginner & $6 / 6(100)$ & $5 / 6(83)$ & $>.999$ \\
\hline Intermediate & $4 / 4(100)$ & $2 / 4(50)$ & .426 \\
\hline Expert & $3 / 4(75)$ & $3 / 4(75)$ & $>.999$ \\
\hline Number of attempts & $1(1-3)$ & $2(1-3)$ & .035 \\
\hline Beginner & $1(1-1)$ & $2.5(1-3)$ & .027 \\
\hline Intermediate & $1.5(1-3)$ & $1.5(1-3)$ & $>.999$ \\
\hline Expert & $1(1-2)$ & $2.5(1-3)$ & .161 \\
\hline Time to access in seconds & $85(31-398)$ & $255(34-480)$ & .009 \\
\hline Beginner & $85(42-164)$ & $368(107-453)$ & .013 \\
\hline Intermediate & $114(65-349)$ & $205(34-480)$ & .665 \\
\hline Expert & $62(31-398)$ & $133(84-444)$ & .194 \\
\hline Fluoroscopy time in seconds* & $1(1-1)$ & $94(12-252)$ & $<.001$ \\
\hline Beginner & $1(1-1)$ & $102(60-168)$ & .003 \\
\hline Intermediate & $1(1-1)$ & $93(12-126)$ & .021 \\
\hline Expert & $1(1-1)$ & $94(42-252)$ & .032 \\
\hline
\end{tabular}

Median time until achieving successful access was shorter with EM-Access compared to FG-Access ( 85 vs 255 seconds, $P<.01$, Table 1 ). This was true of the overall cohort as well as within each skill level (Table 1). Time to access decreased with increasing skill 
level in the FG-Access group. This was not the case for EM-Access where the median time was much closer between skill levels (Table 1).

Fluoroscopy time for attempting access was higher in FG-Access compared to EMAccess (Table 1). This does not take into account fluoroscopy time for achieving retrograde access or positioning the ureteroscope into the desired calyx which was common to both the FG and EM attempts. Calyceal distribution for targeted calyces included 6 upper calyces, 4 middle calyces, and 4 lower calyces. EM-Access was obtained faster for each renal location relative to FG-access (Table 2).

Table 2. Access time comparison between electromagnetic (EM) and fluoroscopic (FG) guided access based on renal calyceal location

\begin{tabular}{lrlc}
\hline & \multicolumn{1}{l}{$\begin{array}{l}\text { EM-Access } \\
\text { (sec) }\end{array}$} & $\begin{array}{l}\text { FG-Access } \\
\text { (sec) }\end{array}$ & P Value \\
Upper Calyx & $73(42-156)$ & $232(107-453)$ & .008 \\
Middle Calyx & $79(31-137)$ & $164(84-480)$ & .194 \\
Lower Calyx & $256(52-398)$ & $394(34-453)$ & .665 \\
\hline
\end{tabular}

Values are medians (range). $P$ values were calculated using Mann-Whitney $U$ test (continuous data).

\section{DISCUSSION}

We found that EM-Access performed favorably relative to FG-Access among a cohort of urologists and trainees with various skill levels. Rates of successful access were no different between groups but successful EM-Access was performed more quickly, with fewer attempts, and less radiation. Notably, performance among beginners with little to no prior experience participating in PCNL surgery had nearly equivalent performance relative to experts with similar success rates, number of attempts, and time to obtaining access. The same was not true for FG-access where there were comparable success rates but a quicker time to access with increasing experience level. The current EM technology provided quicker access to all renal calyceal locations as compared to FGAccess. However, in the EM-Access group, the median time to access lower renal calyces was significantly higher compared to the median time to access upper or middle calyces 
(Table 2). We attribute this observation to a known software limitation in imposing a virtual access tract that is colinear with ureteroscope. Plans are being made to improve the EM software and potentially decrease the time necessary to access lower renal calyces with EM-Access.

The ability to obtain one's own access during PCNL is widely recognized to be a useful skill; yet, appears to be underutilized in clinical practice. In fact, recent estimates from large data sets in the United States and United Kingdom suggest that urologists obtain their own PCNL access in only a minority of cases. Speed et al found that among over 40,000 PCNL's from the United States performed between 2003 and 2015, urologists only obtained access in $17 \%$ of cases. ${ }^{1}$ Armitage et al reviewed over 5000 PCNL cases from a UK PCNL registry and found that urologists only obtained access $34 \%$ of the time. $\underline{2}$

While it has been well demonstrated that PCNL success can be achieved whether radiologists or urologists are the ones obtaining access, there are unique benefits of being able to obtain one's own access as a urologist. Among the numerous advantages are greater surgeon autonomy with less reliance on colleagues, improved scheduling logistics both in terms of surgeon availability as well as reservation of the operating room and/or interventional radiology suite, and increased procedural flexibility in the event the initial access is insufficient to completely treat the stone. Ultimately, PCNL remains a relatively unique procedure in the field of urologic surgery in the sense that it is so commonly performed in conjunction with specialists of another discipline. One is thus left to wonder why this is the case when urologists have become so facile and independent with nearly every other surgical treatment commonly offered.

The most likely explanation for why urologists so frequently work in conjunction with radiologists to perform percutaneous access is a lack of skill in obtaining access independently. There have been numerous attempts to estimate the number of cases necessary to achieve proficiency in PCNL and access with reported case numbers ranging from 25 to 60 cases regardless of whether fluoroscopic guidance or ultrasound guidance is being used. $\underline{3}, \underline{4}, \underline{5}, \underline{6}$ Achieving such proficiency during residency is challenging as not all training programs necessarily have faculty who perform this procedure. In fact, in 2011 fewer than half $(47 \%)$ of graduating US chief residents 
indicated that PCNL access was routinely obtained by urologists at their institution. $\underline{-}$ Opportunities to pick up these skills in practice are limited as well as PCNL has become a highly regionalized procedure. A 2014 review of case logs from certifying and recertifying urologists found that only $6 \%$ performed $>10$ PCNLs during the prior 6 months and urologist obtained access only occurred in $20 \%$ of these cases. $\stackrel{8}{-}$

Additional concerns with existing PCNL access techniques exist as well. Fluoroscopic guided access carries with it a risk of exposure to ionizing radiation, particularly at the early end of the learning curve where fluoroscopy times are known to be greater. -3 Ultrasound guided access on the other hand is subject to suboptimal image acquisition depending on available ultrasound machinery as well as patient body habitus and anatomy. - EM guidance offers advantages of both of these techniques. Similar to fluoroscopy, the graphical user interface provides a wide field with discrete image representation of the needle and the target that is independent of patient factors. Like ultrasound however, it allows the surgeon to visualize the needle and target continuously and align the access in real time without excess exposure to ionizing radiation.

Interest in EM-Access is growing but data remain sparse with no currently available commercial products available for this purpose. The first experience with EM-Access was described by Huber et al in 2011 using the Aurora EM tracking system (Northern Digital Inc., Waterloo, Ontario, Canada) where they reported a single surgeon experience in a porcine model with high accuracy of puncture (91\% first attempt and $100 \%$ second attempt). $\frac{10}{}$ In 2013 Rodrigues et al demonstrated similar success using this system to perform EM-access to various points in the kidney and ureter of a porcine model. A single experienced surgeon and single resident performed all of the punctures with no difference in success rates between the 2.11 The same group more recently demonstrated feasibility of EM-access among a series of 10 patients undergoing PCNL by a single experienced surgeon. All 10 punctures were performed with EM guidance alone on the first attempt. 12 Each of the aforementioned studies of EM-access noted a reduced learning curve as one of the potential advantages of this approach. Our findings are the first to corroborate this in a formal sense both in comparison of outcomes between different surgeon experience levels as well as in comparison to an existing alternative technique for PCNL access. 
Our findings must be viewed in the context of several limitations. First, we did not compare EM-Access to ultrasound access; thus, we cannot comment on how these 2 modalities compare to one another. Our goal was to compare the new technology to fluoroscopy which is our existing standard method of access and the more common approach on a worldwide scale ( $87 \%$ of cases according to a global PCNL database). $\frac{13}{}$ Additionally, endoscopic assistance was required in all cases to deliver the EM targeting beacon to the chosen calyx. While endoscopic combined intrarenal surgery has become more common for PCNL this approach does have several limitations and is not feasible for all cases including those with lower urinary tract reconstruction, staghorn calculi, and calyceal diverticuli. Ultimately, the ability to pass the EM beacon alone without requiring a ureteroscope could increase the applicability of this approach to even these challenges scenarios; however, this was not able to be tested at this time. We also performed all renal punctures with the pigs in the prone position. In theory, the EM tracking should be equally efficacious in the supine position; however, we are unable to conclusively state this with the data obtained. Skin to stone distance is another variable that could theoretically affect performance. From a technologic perspective the EM generator should be able to identify EM enabled items within a range of $50 \mathrm{~cm}$ however we are unable to comment on whether increasing skin to stone and/or target distance could affect performance at this time as all the pigs in this study were between 50 and $70 \mathrm{~kg}$.

Another limitation is that the porcine model used during this study may not be perfectly analogous to human kidneys; however, if anything this would have made the access more difficult as porcine kidneys tend to be smaller in size with more complex and narrow renal pelviceal anatomy. Finally, a larger sample size could have produced a more accurate estimation of the study parameters but was limited from a practicality perspective. Further clinical studies among patients with nephrolithiasis would be one way to obtain more data but will require additional regulatory approval.

\section{CONCLUSION}

EM-Access performed favorably relative to FG-Access. Beginners were able to obtain access with equal success rates and speeds compared to experts with minimal radiation. Future studies should be directed at studying whether similar success rates can be 
achieved in the early learning curve of trainees and urologists in practice. If confirmed, such technology could ultimately help increase urologist obtained access for PCNL in clinical practice.

\section{REFERENCES}

1. Speed, J. M., Wang, Y., Leow, J. J., Bhojani, N., Trinh, Q.-D., Chang, S. L., \& Korets, R. (2017). The Effect of Physician Specialty Obtaining Access for Percutaneous Nephrolithotomy on Perioperative Costs and Outcomes. Journal of Endourology, 31(11), 1152-1156. https://doi.org/10.1089/end.2017.0441

2. Armitage, J. N., Withington, J., Fowler, S., Finch, W. J. G., Burgess, N. A., Irving, S. O., Glass, J., \& Wiseman, O. J. (2017). Percutaneous nephrolithotomy access by urologist or interventional radiologist: Practice and outcomes in the UK. BJU International, 119(6), 913-918. https://doi.org/10.1111/bju.13817

3. Allen, D., O'Brien, T., Tiptaft, R., \& Glass, J. (2005). Defining the Learning Curve for Percutaneous Nephrolithotomy. Journal of Endourology, 19(3), 279-282. https://doi.org/10.1089/end.2005.19.279

4. Ziaee, S. A. M., Sichani, M. M., Kashi, A. H., \& Samzadeh, M. (2010). Evaluation of the Learning Curve for Percutaneous Nephrolithotomy. Urology Journal, 7(4), 226-231. https://doi.org/10.22037/uj.v7i4.818

5. Tanriverdi, O., Boylu, U., Kendirci, M., Kadihasanoglu, M., Horasanli, K., \& Miroglu, C. (2007). The Learning Curve in the Training of Percutaneous Nephrolithotomy. European Urology, 52(1), 206-212. https://doi.org/10.1016/j.eururo.2007.01.001

6. Song, Y., Ma, Y., Song, Y., \& Fei, X. (2015). Evaluating the Learning Curve for Percutaneous Nephrolithotomy under Total Ultrasound Guidance. PLOS ONE, 10(8), e0132986. https://doi.org/10.1371/journal.pone.0132986

7. Spann, A., Poteet, J., Hyatt, D., Chiles, L., DeSouza, R., \& Venable, D. (2011). Safe and Effective Obtainment of Access for Percutaneous Nephrolithotomy by Urologists: The Louisiana State University Experience. Journal of Endourology, 25(9), 1421-1425. https://doi.org/10.1089/end.2010.0643 
8. Jayram, G., \& Matlaga, B. R. (2014). Contemporary Practice Patterns Associated with Percutaneous Nephrolithotomy Among Certifying Urologists. Journal of Endourology, 28(11), 1304-1307. https://doi.org/10.1089/end.2014.0465

9. Usawachintachit, M., Masic, S., Chang, H. C., Allen, I. E., \& Chi, T. (2016). Ultrasound Guidance to Assist Percutaneous Nephrolithotomy Reduces Radiation Exposure in Obese Patients. Urology, 98, 32-38. https://doi.org/10.1016/j.urology.2016.04.012

10. Huber, J., Wegner, I., Meinzer, H.-P., Hallscheidt, P., Hadaschik, B., Pahernik, S., \& Hohenfellner, M. (2011). Navigated renal access using electromagnetic tracking: An initial experience. Surgical Endoscopy, 25(4), 1307-1312. https://doi.org/10.1007/s00464-010$\underline{1338-\mathrm{x}}$

11. Rodrigues Pedro L., Vilaça João L., Oliveira Carlos, Cicione Antonio, Rassweiler Jens, Fonseca Jaime, Rodrigues Nuno F., Correia-Pinto Jorge, \& Lima Estevão. (2013). Collecting System Percutaneous Access Using Real-Time Tracking Sensors: First Pig Model In Vivo Experience. Journal of Urology, 190(5), 1932-1937. https://doi.org/10.1016/j.juro.2013.05.042

12.Lima, E., Rodrigues, P. L., Mota, P., Carvalho, N., Dias, E., Correia-Pinto, J., Autorino, R., \& Vilaça, J. L. (2017). Ureteroscopy-assisted Percutaneous Kidney Access Made Easy: First Clinical Experience with a Novel Navigation System Using Electromagnetic Guidance (IDEAL Stage 1). European Urology, 72(4), 610-616. https://doi.org/10.1016/j.eururo.2017.03.011

13. Andonian, S., Scoffone, C. M., Louie, M. K., Gross, A. J., Grabe, M., Daels, F. P. J., Shah, H. N., \& de la Rosette, on behalf of the C. P. S. G., Jean J. M. C. H. (2012). Does Imaging Modality Used for Percutaneous Renal Access Make a Difference? A Matched Case Analysis. Journal of Endourology, 27(1), 24-28. https://doi.org/10.1089/end.2012.0347

\section{EDITORIAL COMMENT}

The authors report their initial experience with electromagnetic (EM) guidance to facilitate percutaneous puncture in a porcine model and compare it to traditional fluoroscopic guided access with participants who had a range of prior experience with percutaneous puncture. Overall the results appear promising with shorter access times and a reduction in the overall number of puncture attempts. EM guidance also provides the important 
benefit of reduced fluoroscopic radiation exposure to the patient, surgeon, and room staff. These results suggest the technology holds promise however I am not yet convinced that this is going to be the solution to the problem of urologists not obtaining their own access, especially in the United States and United Kingdom. As discussed in the manuscript, it is estimated only $17 \%$ of PCNL cases in the United States have access performed by a urologist while in the United Kingdom that number is high, but still only 34\%.1,2 Further, it has been reported that only $6 \%$ of US urologists are "high volume" PCNL surgeons, defined by the relatively low bar of greater than 10 cases in a 6-month period. 3 While one may propose that EM guidance may shorten the learning curve for percutaneous access and help to improve upon these very low numbers, I would suggest that EM guidance may add significant cost and complexity to a procedure that can be done very effectively with existing fluoroscopic and ultrasonic technology. The focus needs to be on training our residents how to achieve access, a skill that can be acquired with good proficiency in about 24 cases. 4 While EM guided access may hold promise, we must focus on the real issue at hand, the training of our residents. Bodo E. Knudsen, The OSU Comprehensive Kidney Stone Program, Henry A. Wise II Endowed Chair in Urology, Department of Urology, The Ohio State University Wexner Medical Center, Columbus, $\mathrm{OH}$

\section{References}

1. Armitage JN, Withington J, Fowler $\mathrm{S}$, et al. Percutaneous nephrolithotomy access by urologist or interventional radiologist: practice and outcomes in the UK. BJU Int. 2017;119:913-918. 2

2. . Speed JM, Wang Y, Leow JJ, et al. The Effect of Physician Specialty Obtaining Access for Percutaneous Nephrolithotomy on Perioperative Costs and Outcomes. J Endourol. 2017;31:1152-1156.

3. Jayram G, Matlaga BR. Contemporary practice patterns associated with percutaneous nephrolithotomy among certifying urologists. J Endourol. 2014;28:1304-1307.

4. de la Rosette JJ, Laguna MP, Rassweiler JJ, Conort P. Training in percutaneous nephrolithotomy-a critical review. Eur Urol. 2008;54: 994-1001.

\section{AUTHOR REPLY}


We appreciate the sentiments and concerns expressed in the aforementioned editorial and agree completely that the training of resident physicians in PCNL access is of paramount importance. However, we believe that there is also substantial room for the introduction of new technologies to facilitate this goal. Fluoroscopic and ultrasonic technology for PCNL access has existed, largely unchanged, for many years, providing an ample amount of time for urologists to have become facile in obtaining their own access. Nonetheless, a substantial minority of urologists choose not to, begging the question, why not? One of the most common reasons is that many urologists do not feel confident using existing techniques for this purpose.1 Certainly, greater exposure during residency would be useful; however, with the paucity of high-volume PCNL surgeons and low percentage of urologists who routinely obtain their own access it is likely that not all residents have opportunities to master such skills during training. Adjuncts to residency training for PCNL are becoming more popular including a growing number of practical and hands on courses.2 Similarly, there is work being done to integrate virtual and augmented reality into training models 3,4 via simulation that might prove useful in this area. In the meantime, exploring new technologies that might simplify the process of obtaining PCNL access should be encouraged as well. Concerns about potential extra cost and complexity of any new technology is warranted; however, one must also be cognizant of the extra cost and complexity of PCNL access when not obtained by a urologist. For the surgeon this includes coordination of schedules with another subspecialty physician. For the patient, this may mean having to undergo 2 separate procedures as it remains common practice in many institutions to have access placed preoperatively to the PCNL by a separate physician, in a separate part of the hospital, at a separate time. Ultimately, more clinical data and real-life experience will be necessary to determine whether EM guidance proves useful for PCNL. In the interim this does not mean abandoning efforts to educate trainees. One hopes that ultimately these goals are not mutually exclusive. Michael S. Borofsky, Marcelino E. Rivera, Casey A. Dauw, Amy E. Krambeck, James E. Lingeman, University of Minnesota, Minneapolis, MN; Mayo Clinic, Rochester, MN; Indiana University, Indianapolis, IN; University of Michigan, Ann Arbor, Ml.

\section{References}


1. Bird VG, Fallon $B$, Winfield HN. Practice patterns in the treatment of large renal stones. $J$ Endourol. 2003;17:355-363.

2. Noureldin YA, Hoenig DM, Zhao P, et al. Incorporation of the fluoroless C-Arm trainer at the American Urological Association hands on training percutaneous renal access. World J Urol. 2018;36:1149- 1155.

3. Rassweiler-Seyfried MC, Rassweiler JJ, Weiss C, et al. iPad-assisted percutaneous nephrolithotomy (PCNL): a matched pair analysis compared to standard PCNL. World $\mathrm{J}$ Urol. 2019. Epub ahead of print.

4. Ghazi A, Campbell T, Melnyk R, et al. Validation of a full-immersion simulation platform for percutaneous nephrolithotomy using threedimensional printing technology. J Endourol. 2017;31:1314-1320. 